\title{
Análise comparativa dos resultados funcionais obtidos em 100 artroplastias totais do joelho pela fisioterapia convencional isolada ou associada à movimentação passiva contínua
}

\author{
Eliane Machado Máximo* \\ Danilo Masiero** \\ Luiz Aurélio Mestriner ***
}

\section{RESUMO}

Estudo prospectivo de 85 pacientes submetidos a 100 artroplastias totais dos joelhos (15 bilaterais) no período compreendido entre junho de 1991 a junho de 1994.

Os pacientes foram distribuídos em dois grupos. O grupo I, constituído de 44 pacientes (50 joelhos), foi submetido à fisioterapia convencional isolada e o grupo II de 45 pacientes (50 joelhos) à fisioterapia convencional associada à MPC (movimentação passiva contínua). O objetivo foi estabelecer possíveis diferenças entre os dois tipos de tratamento fisioterápico com relação ao tempo mínimo necessário para atingir 60 graus de flexão, tempo de hospitalização, variação percentual do arco de movimento pré e pós-operatório e condições da ferida operatória. Foi também analisada a evolução do arco de movimento por ocasião da alta hospitalar, e aos três, seis, nove e doze meses. Não houve diferença estatisticamente significante em relação aos parâmetros analisados.

\section{ABSTRACT}

Eighty - five patients underwent 100 knee arthroplasties utilizing the condylar-type prosthesis performed between 1991 and 1994 have been analized. The patients were separated in two groups: group I consisted of 44 patients (50 knees) was managed by physical therapy only and the group II of 45 patients (50 knees) submited to physical therapy associated to continuous passive motion (CPM) in order to compare the difference between two rehabilitation protocols. The study compares the time required to obtain 60 degrees of knee flexion, the length of hospital stay, the percentage of pre and postoperative range of motion and wound healing complications. The results were analysed before discharge and the range of motion was available in three, six, nine and twelve months. The results showed no statistically significant differences between the groups.

Trabalho realizado na Disciplina de Fisiatria, Setor de Fisioterapia do Departanento de Ortopedia e Traumatologia da UNIFESP/EPM (Serviço do Prof. Dr. José Laredo Filho).

Mestre em Ciências da Saúde - Área de Reabilitação do Departamento de Ortopedia e Traumatologia da UNIFESP - Escola Paulista de Medicina

** Professor Adjunto - Doutor e chefe da Disciplina de Fisiatria do Departamento de Ortopedia e Traumatologia da UNIFESP - Escola Paulista de Medicina

*** Professor-Adjunto - Doutor do Departamento de Ortopedia e Traumatologia da UNIFESP-Escola Paulista de Medicina

Endereço para correspondência:

Rua dos Açores, 310

CEP 04032-060 - São Paulo / SP 


\section{Introdução}

A recuperação do arco de movimento articular tem sido uma preocupação constante não apenas para o cirurgião, mas também para o terapêuta reabilitador, sempre no sentido de buscar a independência funcional do paciente.

Salter et. al. ${ }^{24}$, mostrando preocupação com a reabilitação do movimento após as lesões articulares, desenvolveram um trabalho experimental em que a movimentação contínua é utilizada com a finalidade de estimular a reparação tecidual e, posteriormente, utilizando metodologia semelhante Salter, Bell, Keeley ${ }^{22}$ e Salter et. al. ${ }^{23}$, analisam também a influência da técnica para o tratamento coadjuvante das artrites e fraturas articulares.

A artoplastia do joelho é uma cirurgia que determina grande comprometimento tecidual intra e extra articular e como tal, passível de aderências pós-operatórias que podem determinar a rigidez articular.

Durante a década de 1980, a preocupação com a evolução funcional após artroplastia do joelho ficou evidente a partir dos trabalhos de Coutts et. al. $^{2}$, Davis ${ }^{4}$ e tantos outros ${ }^{17,18}$ até Nadler, Malanga, Zimmerman ${ }^{16}$, cujos objetivos principais eram os de estabelecer as vantagens de movimentação passiva contínua sobre a fisioterapia convencional.

Considerando algumas controvérsias, motivamo-nos a analisar, comparativamente, os resultados obtidos, utilizando a fisioterapia convencional de rotina ou associada à movimentação passiva contínua.

Os objetivos básicos deste estudo foram os de analisar os possíveis benefícios da movimentação passiva contínua, em especial com relação à evolução e permanência hospitalar, fatores esses também diretamente relacionados aos problemas econômicos, evidentes em nosso meio.

\section{Material e método}

Neste estudo são analisados, prospectivamente, 85 pacientes submetidos a 100 artroplastias totais dos joelhos (15 bilaterais), no período entre junho de 1991 a junho de 1994. Todos os pacientes estão registrados no Departamento de Ortopedia e Traumatologia da UNIFESP - Universidade Federal de São Paulo - Escola Paulista de Medicina - Serviço do Prof. Dr. José Laredo Filho. Todas as artroplastias foram primárias e as bilaterais foram realizadas em tempos diferentes. As próteses utilizadas foram as de superfície ou condilares do tipo Whiteside (59 joelhos) e "Condilar Total"(Baumer), (41 joelhos).
A idade dos pacientes variou de 32 a 83 anos com média de 66,03 anos. Em relação à artrite reumatóide (AR) a idade variou de 32 a 74 anos com média de 56,45 anos. Para a osteoartrose (AO) a idade variou de 50 a 83 anos, com média de 69,29 anos. Um paciente (75 anos) era portador de osteonecrose (ON). Sessenta e nove pacientes $(81,17 \%)$ eram do sexo feminino e $16(18,82 \%)$ do masculino. Setenta e três pacientes $(85,88 \%)$ eram brancos e $12(14,11 \%)$ não brancos.

Quanto ao diagnóstico, 22 pacientes $(25,88 \%)$ e 30 joelhos (30\%) eram portadores de AR ( 8 bilaterais). Sessenta e dois pacientes $(72,94 \%)$ e 69 joelhos $(69 \%)$ eram portadores de OA (7 bilaterais). Um paciente $(1,17 \%)$ e 1 joelho $(1 \%)$ eram portadores de osteonecrose. Com relação ao lado, 49 operações $(49 \%)$ foram sobre o joelho direito e 51 $(51 \%)$ sobre o esquerdo.

Considerando o diagnóstico, nos portadores de AR, 14 joelhos apresentavam desvio em valgo $(46,66 \%), 8$ em varo $(26,66 \%)$ e 8 apresentavam alinhamento neutro $(26,66 \%)$. Em relação à $O A$, 11 apresentavam desvio em valgo (15,94\%), $30 \mathrm{em}$ varo $(43,47 \%)$ e 28 apresentavam alinhamento neutro $(40,57 \%)$. O paciente portador de $\mathrm{ON}$ apresentava alinhamento neutro.

Com relação à $\mathrm{AR}$, o arco de movimento préoperatório variou de 30 a 140 graus com média de 95,66 graus, sendo que a atitude de flexão variou de 5 a 50 graus com média de 12,83 graus e a flexão total variou de 70 a 130 graus com média de 108,5 graus. Com relação à $\mathrm{OA}$, o arco de movimento variou de 30 a 140 com média de 104,92 graus, sendo que a atitude de flexão variou de 5 a 30 graus com média de 3,40 graus e a flexão total variou de 30 a 140 graus com média de 107,89 graus. O paciente portador de $\mathrm{ON}$ apresentava arco de movimento de 130 graus.

O tempo de imobilização pós-operatório variou de 2 a 8 dias com média de 4,22 dias, independente do diagnóstico.

Com relação ao tipo de fisioterapia, 50 joelhos $(50 \%)$ foram submetidos à fisioterapia convencional e $50(50 \%)$ à mesma técnica associada à MPC (movimentação passiva contínua). Na AR o período de internação variou de 8 a 29 dias com média de 3,46 dias e na OA de 6 a 79 dias com média de 8,88 dias. O portador de ON esteve internado por 15 dias.

Durante o pós-operatório imediato até a retirada do enfaixamento, os pacientes eram estimulados para exercícios isométricos do quadríceps, visando manter o trofismo muscular. Além disto, estimulavase a movimentação ativa do tornozelo mantendo assim as condições circulatórias bem como a movimentação ativo-assistida do quadril (flexão e abdução) com o objetivo de ganhar força muscular. 
Com a retirada da imobilização, os pacientes eram sorteados e divididos em dois grupos. O Grupo I, constituído de 44 pacientes (50 joelhos) foi submetido à fisioterapia convencional através da movimentação ativo-assistida (Fig. 1-A) do joelho utilizando, inclusive, o auxílio de um sistema de polias adaptadas ao leito (Fig. 1-B), exercícios isotônicos para a musculatura do quadril e joelho durante 1 hora e treino de marcha com andador. $\mathrm{O}$ Grupo II, constituído de 45 pacientes, (50 joelhos) foi submetido à fisioterapia convencional associada à utilização da movimentação passiva contínua (MPC) (Fig. 2), por 3 horas diárias ${ }^{8}$, sendo a máquina programada para 10 graus iniciais de flexão, em velocidade média, com progressão a cada 24 horas conforme o grau de tolerabilidade do paciente.

Os resultados foram analisados na alta hospitalar considerando a flexão mínima como de 60 graus, tempo de hospitalização, porcentagem de modificação do arco de movimento pré e pós-operatório e condições locais que incluiam: edema superficial, derrame articular e complicações de pele tais como secreção da ferida e deiscência de sutura.

É importante assinalar que 60 graus de flexão era o objetivo a ser atingido para que os pacientes pudessem ser liberados para o tratamento fisioterápico de nível ambulatorial. A necessidade de manter a internação por tempo mais prolongado ficava a critério do cirurgião.

Para avaliar as possíveis diferenças entre os mesmos parâmetros dos dois grupos estudados, utilizamos os testes estatísticos não paramétricos

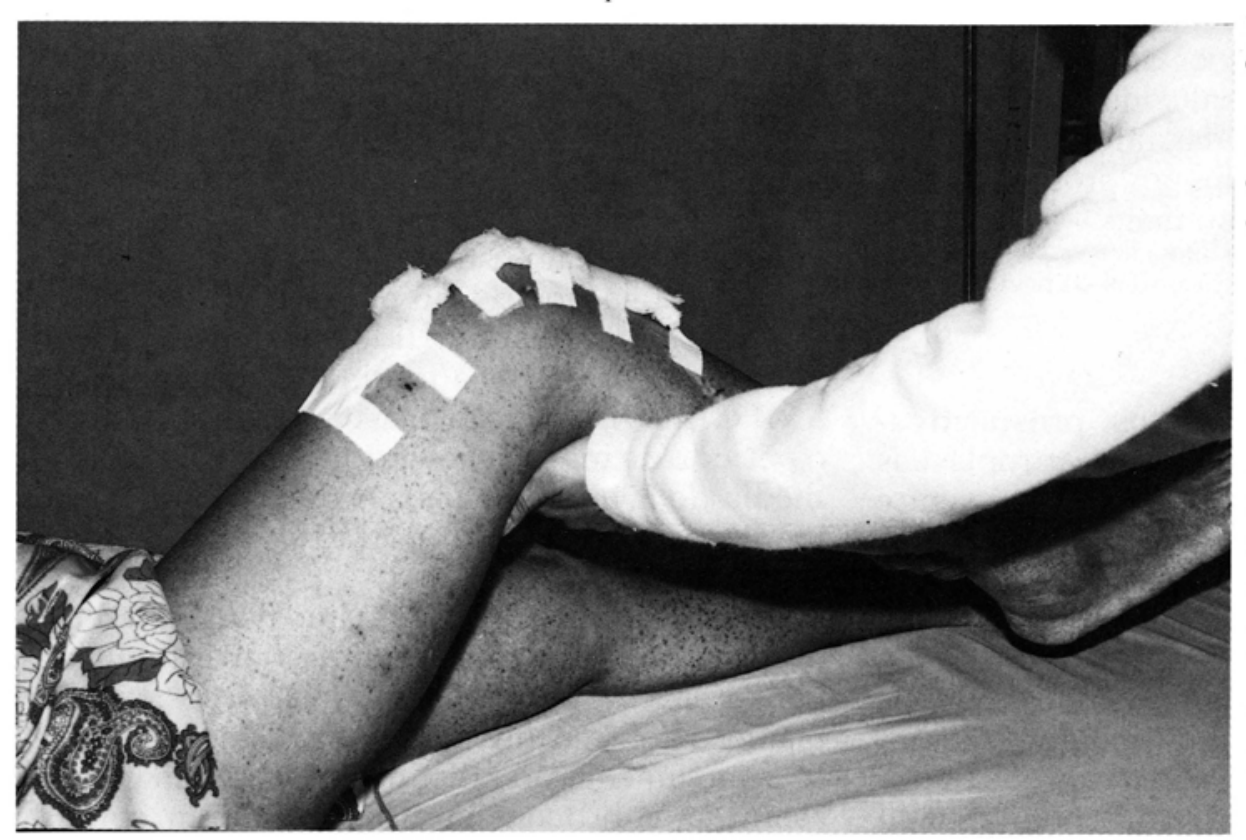

Figura 1-A

Fisioterapia ativo-assistida de Kruskal-Wallis ${ }^{25}$. Foram analisados em especial os parâmetros: tempo decorrido para o joelho atingir 60 graus de flexão, tempo de hospitalização e percentual do arco de movimento pré e pós-operatório. Além disto, foram analisados as possíveis intercorrências pós-operatórias bem como o arco de movimento aos três, seis, nove e doze meses.

\section{Resultados}

O tempo decorrido para que o joelho submetido à artroplastia total atingisse 60 graus de flexão, foi em média de 2,34 dias para o grupo I e de 2,68 dias para o grupo II, diferença esta sem significância estatística, considerando os dois grupos.

O tempo de hospitalização não mostrou diferença estatisticamente significante entre os dois tipos de reabilitação utilizados, independente do diagnóstico considerado (AR ou OA). Do mesmo modo, os arcos de movimento pré e pós-operatórios não mostraram diferenças percentuais estatisticamente significantes, considerando os dois tipos de reabilitação.

Não foi possível estabelecer uma correlação estatisticamente significante das intercorrências pós-operatórias com os métodos da reabilitação empregados, embora fatores como: treinamento do terapeuta, dificuldade de manuseio da máquina e condições do paciente possam ser determinantes.

A análise do arco de movimento aos três, seis, nove e doze meses de pósoperatório, mostrou uma tendência à melhora funcional independente do tipo de reabilitação considerada.

\section{Discussão}

A artroplastia total, talvez uma das cirurgias mais radicais sobre a articulação do joelho, apresentou um desenvolvimento muito importante a partir da década de 70 na Inglaterra (Freeman) e Estados Unidos (Insall).

Desde então começaram a aparecer os problemas relacionados ao 
método, tais como a escolha da prótese e a técnica cirúrgica ideal, bem como as complicações pósoperatórias entre as quais, infecção, instabilidade, cicatrização da ferida, trombose venosa profunda e a própria rigidez articular são as mais importantes.

As técnicas de fisioterapia passiva e ativa assistidas, têm sido constantemente desenvolvidas sempre no sentido de se obterem melhores resultados funcionais, sem esquecer do fator biológico no processo da reabilitação articular. O fisioterapeuta deve conhecer todos os fatores mecânicos e biológicos envolvidos

na recuperação da amplitude de movimento do joelho, para que possa estabelecer a exata programação de cada etapa do tratamento.

A indicação de uma artroplastia do joelho deve evidentemente partir da premissa de que, o principal estaqueador, o quadríceps, tenha a função adequada para o trabalho de reabilitação. Sendo assim, admite-se que a principal meta, além da reabilitação muscular, é a recuperação da amplitude de movimento.

O que seria ideal? A reabilitação por fisiotera-

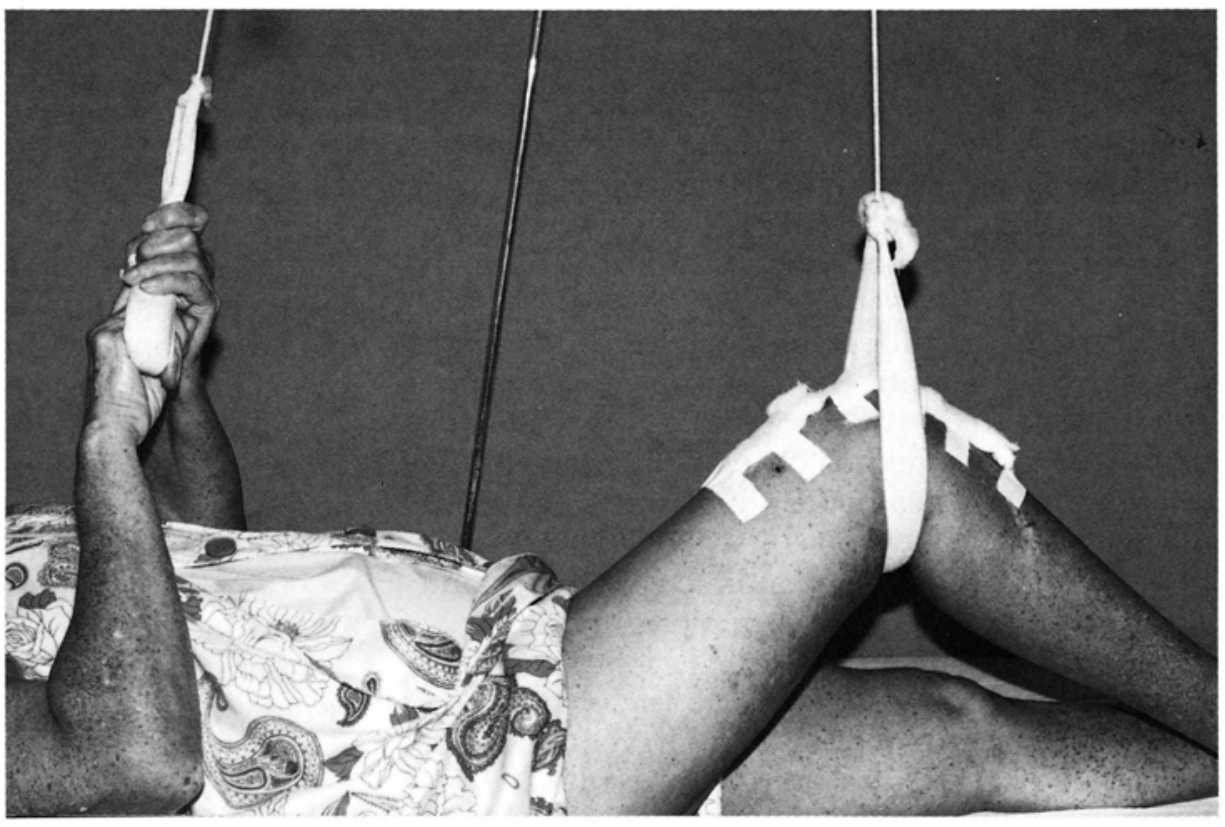

Figura 1-B

Fisioterapia ativo-assistida com auxilio de polias

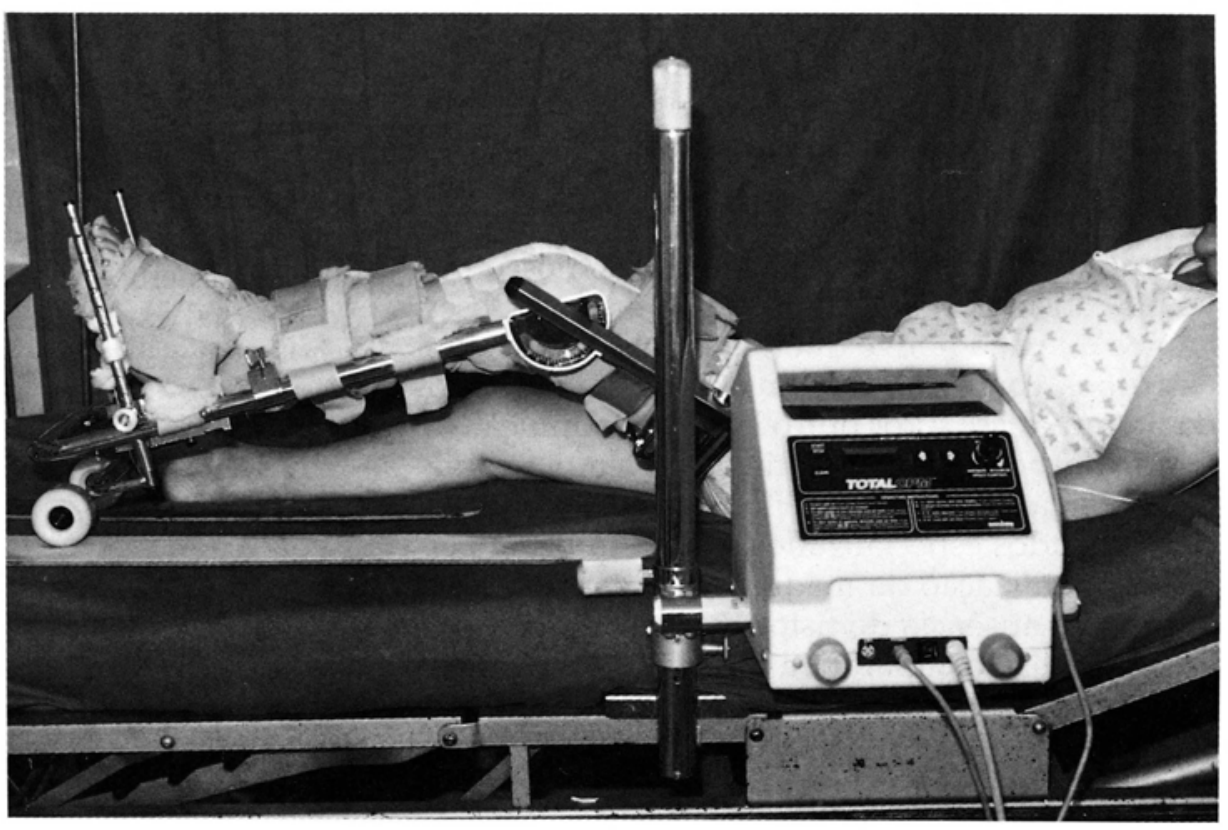

Figura 2

Movimentação passiva continua em flexão pia convencional ativoassistida? A movimentação passiva contínua ? Ambos os métodos?

Independente dos estudos da influência da movimentação passiva contínua, citados na literatura, considerando alguns parâmetros tais como: a evolução da ferida cirúrgica ${ }^{5,7,10,11,15,21,23,27}$, incidência e evolução da trombose venosa profunda $a^{5,13,14,26}$ e sangramento pós-operatório ${ }^{1,2,3,7}$, imaginamos que se a movimentação passiva contínua apresentasse vantagens quanto ao tempo de hospitalização pós-operatória, isto por si só justificaria

a utilização de máquinas em todos os pacientes envolvidos em artroplastia do joelho. Por outro lado, um fator diretamente ligado ao anterior, seria a recuperação da independência funcional, ou seja, a reabilitação para as mínimas atividades da vida diária.

A observação diária dos pacientes operados nos levou à idéia de que, um arco de movimento que estivesse compreendido entre 10 a 60 graus, permitia supor que a evolução posterior estaria assegurada. 
O nosso material, constituído de pacientes portadores de AR e OA, permitiu um estudo comparativo da evolução hospitalar do arco de movimento considerando o tipo de fisioterapia empregado, além de possibilitar a avaliação do comportamento do paciente reumatóide e do artrósico,

Quando consideramos o tempo decorrido para atingir a meta de 60 graus, procuramos correlacionar o tipo de fisioterapia com o diagnóstico e o sexo e pudemos observar que não houve diferença estatisticamente significante nos resultados obtidos.

Além disto, o fator econômico era importante e não poderíamos aguardar maior grau de flexão para permitir a alta do paciente. Este fato foi muito bem observado por Ritter et. al. ${ }^{19}$ que consideraram o preço excessivo da aparelhagem, além do difícil manuseio. Outra preocupação que tivemos com movimentação passiva contínua foi em relação à evolução da ferida. Procuramos então, fazer a progressão do ângulo proporcionado pela máquina de maneira leve pois, como observou Goletz \& $\mathrm{Henry}^{7}$, que consideraram como meta os 90 graus de flexão, se esta fosse atingida antes do sexto dia, problemas cicatriciais poderiam ocorrer.

Embora vários fatores possam influir no período total da hospitalização pós-operatória, pudemos também comparar este tempo em nosso material, considerando o diagnóstico e sexo. Observamos que não houve diferença significante entre os grupos, concordando com os resultados obtidos da literatura ${ }^{12,16,20}$. Por outro lado, a diminuição do tempo de hospitalização utilizando a movimentação passiva contínua ${ }^{1,3,4,5,6,7,10,11}$, estaria relacionada com cuidados especializados de enfermagem permitindo utilização da máquina por maior tempo, mesmo na ausência do fisioterapeuta.

A análise comparativa do arco de movimento pré e pós-operatório deveria levar em consideração o tipo de prótese utilizada, uma vez que o grau de flexão final obtido muitas vezes depende do desenho protético. Deste modo, por estarmos trabalhando com próteses de características mecânicas pouco diferentes, preferimos a análise percentual do arco de movimento pré e pós-operatório e verificamos que não houve diferença estatisticamente significante tanto no sentido positivo como no negativo, considerando como flexão mínima ideal a de 90 graus.

Ao analisar a evolução funcional em intervalos de tempo mais longos, após a alta hospitalar, pudemos considerar os dados obtidos aos três, seis, nove e doze meses de evolução. Consideramos para isto três parâmetros que julgamos fundamentais para a análise funcional: contratura em flexão, flexão ativa máxima e, é claro, o arco de movimento.
O estudo estatístico destes parâmetros, em ambos os tipos de fisioterapia, em nossa opinião, talvez pudesse merecer críticas, uma vez que não foi possível, no segmento ambulatorial, estabelecer um protocolo comum de trabalho fisioterápico a ser administrado a todos os pacientes. Além disso a diversidade sócioeconômica dos nossos pacientes não garantia uma resposta uniforme à orientação domiciliar. Mesmo assim observamos uma tendência à melhora ao longo do tempo dos parâmetros citados, o que se evidencia pelas médias aritméticas calculadas. Estes dados estão em concordância com a literatura que demonstra, em geral, uma melhora na evolução funcional dos joelhos operados ${ }^{10,11}$

A análise das intercorrências e complicações durante o pós-operatório não teve correlação com os resultados finais.

Como considerações finais, podemos afirmar que uma fisioterapia convencional bem conduzida pode determinar resultados satisfatórios em reabilitação de pacientes submetidos à artroplastia total do joelho. A utilização da movimentação passiva contínua, entretanto, não deve ser descartada e, ao contrário, deve ser estimulada, pois é possível que, a evolução econômico-social possa facilitar a utilização de mais unidades mecânicas, que poderão inclusive permitir melhor treinamento do pessoal especializado. Isto, associado a uma análise de maior número de casos, poderá demonstrar a importância da movimentação passiva contínua nestes pacientes.

\section{Referências bibliográficas}

1. COLWELL, C.W. \& MORRIS, B.A. - The influence of continuous passive motion on the results of total Knee arthroplasty. Clin. Orthop. (276):225-8, 1992.

2. COUTTS, R. D.; KAITA, J.; BARR, R.; MASON, R.; DUBE, R.; AMIEL, D.: WOO, S.L. \&Y.; NICKEL,V. - The role of continuous passive motion in the postoperative rehabilitation of the total knee patient. Orthop. Trans., 6(2): 277-78, 1982.

3. COUTTS, R. D.: SHARP, D. N.: BORDEN, L. S.; BRYAN, R. S.: HUNGERFORD. D. S.; STULBERG. S. D.; THOMAS,W.H.; VOLZ. R. G. - The effect of continuous passive motion on total knee rehabilitation. Orthop. Trans., 7:535, 1983.

4. DAVIS, D. - Continuous passive motion for total knee arthroplasty. Phys. Ther., 64:709, 1984.

5. ECKER, M. L. \& LOTKE, P. A. - Postoperative care of the total knee patient. Orthop. Clin. North Am., 20(1): 55-62. 1989.

6. FISHER, R. L.; KLOTER, K.; BZDYRA, B.; COOPER, J. A. - Continuous passive motion (CPM) following total knee replacement. Conn-Med. 49(8): 498-500, 1985.

7. GOLETZ, T. H. \& HENRY, J.H. - Continuous passive motion after total knee arthroplasty. South. Med.J., 79(9): $1116-20,1986$.

8. GOSE, J.C. - Continuous passive motion in the posto-perative treatment of patients with total knee replacement. A retrospective study. Phys. Ther. 67(1):39-42, 1987.

9. HERANI, M. L. G. - Normas para apresentaçāo de dissertação e teses. São Paulo, BIREME, 1990.45p. 
10. JOHNSON, D. P. - The effect of continuous passive motion on wound healing and joint mobility after knee arthroplasty. J. Bone Jt. Surg., 72-A(3):421-26, 1990.

11. JOHNSON, D. P. \& EASTWOOD, D. M. - Beneficial effects of continuous passive motion after total condylar knee arthroplasty. Ann. R. Coll. Surg. Engl., 74 (6):412-6, 1992

12. KNAPP, \&. \& BASSO, M. - Comparison of two continuous passive motion protocols for patients with total knee implants. Phys. Ther., 67(3):360-63, 1987.

13. LYNCH, J. A.; BAKER, P.L.; POLLY, R. E.; MCLOY, M. T.; SUND, K.; ROUDYBUSH, D. - Continuous passive motion: A prophylaxis for deep venous thrombosis following total knee replacement. Orthop. Trans., 8:400, 1984.

14. LYNCH, A. F.; BOURNE, R. B.; RORABECK, C. H.; RANKIN, R. N.; DONALD. A. - Deep-vein thrombosis and continuous passive motion after total knee arthroplasty. J. Bone Jt. Surg., 70A(1):11-14, 1988.

15. MALONEY, W. J.; SCHURMAN, D. J.; HANGEN, D.; GOODMAN, S. B.: EDWORTHY, S.: BLOCH. D. A. - The influence of continuous passive motion on outcome in total knee arthroplasty. Clin. Orthop., 256:162-68, 1990.

16. NADLER, S. F.; MALANGA, G.A.; ZIMMERMAN, J.R.-Continuous passive motion in the rehabilitation setting. A retrospective study. Am. J. Phys. med. Rehabil., 72 (3):162-5, 1993.

17. NIELSEN, P. T.; RECHNAGEL, K.; NIELSEN, S. E. - No effect of continuous passive motion after arthroplasty of the knee. Acta. Orthop. Scand., 59 (5):580-81, 1988.

18. ROMNESS, D.W. \& RAND, J.A. - The role of continuous passive motion following total knee arthroplasty. Clin. Orthop., 226:34-37, 1988.
19. RITTER, M. A.; GANDOLF, V. S.; HOLSTON, K. S. - Continuous passive motion versus physical therapy in total knee arthroplasty. Clin. Orthop., 244:239-43, 1989.

20. ROMNESS, D. W. \& RAND, J.A. - The role of continuous passive motion following total knee arthroplasty. Clin. Orthop., 226:34-37, 1988.

21. ROYEN, B. J.V.; O'DRISCOLL. S. W.; DHERT, W. J.A.- SALTER, R. B - A comparison of the effects of immobilization and continuous passive motion on surgical wound healing in mature rabbits. Plast. and reconstr. Surg., 78:360-66, 1986

22. SALTER, R. B.; BELL, R. S.; KEELEY, F.W. - The protective effect of continuous passive motion on living articular cartilage in acute septic arthritis: An experimental investigation in the rabbit. Clin. Orthop., 159:223-47, 1981.

23. SALTER, R.B.: CLEMENTS, N.D.; OGILVIE-HARRIS, D.; BOGOCH. E. R.; WONG, D. A.; BELL, R. S.; MINSTER, R.- The healing of articular tissues through passive motion: Essence of the first 10 years of experimental investigation. J. Bone Jt. Surg., 64B: 640, 1982.

24. SALTER, R. B.: SIMMONDS, D. F.: MALCOLM, B. W.; RUMBLE, E. J.: MACMICHAEL, D.: CLEMENTS, N.D. - The biological effect of continuous passive motion on the healing of full-thickness defects in articular cartilage. An experimental investigation in the rabbit. J. Bone Jt. Surg., 62A:1232-51, 1980.

25. SIEGEL, S. - Estadistica no Parametrica. Ed. Trillas, Mexico, 1975. p. 346.

26. VINCE, K. G.; KELLY, M. A.; BECK, J.; INSALL, J. N. - Continuous passive motion after total knee arthroplasty. J. Arthrop., 2(4):281-4, 1987

27. YOUNG, J. S. \& KROLL, M. A. - Continuous passive motion compared to active assisted range of motion. Phys. Ther., 64:721, 1984 